\title{
Classical Limit of Non-Integrable Systems
}

\author{
Mario Castagnino \\ Institutos de Física de Rosario y de Astronomía y Física del Espacio, \\ Casilla de Correos 67, Sucursal 28, 1628 Buenos Aires, Argentina
}

Received on 6 January, 2005

\begin{abstract}
Self-induced decoherence formalism and the corresponding classical limit are extended from quantum inte-
\end{abstract} grable systems to non-integrable ones.

\section{Introduction}

Decoherence was initially considered to be produced by $d e$ structive interference [1]. Later the strategy changed and decoherence was explained as caused by the interaction with an environment [2], but this approach is not conclusive because:

i.- The environment cannot always be defined, e. g. in closed system like the universe.

ii.-There is not a clear definition of the "cut" between the proper system and its environment.

iii.- The definition of the pointer basis is not simple.

So we need a new and complete theory: The self-induced approach [3], based in a new version of destructive interference, which will be explained in this talk in its version for non-integrable systems. The essential idea is that this interference is embodied in Riemann-Lebesgue theorem where it is proved that if $f(\nu) \epsilon \mathbb{L}_{1}$ then

$$
\lim _{t \rightarrow \infty} \int_{-a}^{a} f(\nu) e^{-i \frac{\nu t}{\hbar}} d t=0
$$

If we use this formula in the case when $\nu=\omega-\omega^{\prime}$, where $\omega, \omega^{\prime}$ are the indices of the density operator $\widehat{\rho}$, in such a way that $\nu=0$ corresponds to the diagonal, we obtain a catastrophe, since all diagonal and not diagonal terms would disappear. But, if $f(\nu)=A \delta(\nu)+f_{1}(\nu)$, where now $f_{1}(\nu) \in \mathbb{L}_{1}$, we have

$$
\lim _{t \rightarrow \infty} \int_{-a}^{a} f(\nu) e^{-i \frac{\nu t}{\hbar}} d t=A
$$

and the diagonal terms $\nu=0$ remain while the off-diagonal ones vanish. This is the trick we will use below.

\section{Weyl-Wigner-Moyal mapping}

Let $\mathcal{M}=\mathcal{M}_{\in(\mathcal{N}+\infty)} \equiv \mathbb{R}^{\in(\mathcal{N}+\infty)}$ be the phase space. The functions over $\mathcal{M}$ will be called $f(\phi)$, where $\phi$ symbolizes the coordinates of $\mathcal{M}$

$$
\phi^{a}=\left(q^{1}, \ldots, q^{N+1}, p_{q}^{1}, \ldots, p_{q}^{N+1}\right)
$$

Then the Wigner transform reads

$$
\operatorname{symb} \widehat{f} \stackrel{\circ}{=}(\phi)=\int\langle q+\Delta|\widehat{f}| q-\Delta\rangle e^{i \frac{p \Delta}{\hbar}} d^{N+1} \Delta
$$

where $\widehat{f} \epsilon \widehat{\mathcal{A}}$ and $f(\phi) \epsilon \mathcal{A}$ where $\widehat{\mathcal{A}}$ is the quantum algebra and the classical one is $\mathcal{A}$. We can also introduce the star product

$$
\begin{gathered}
\operatorname{symb}(\widehat{f} \widehat{g})=\operatorname{symb} \widehat{f} * \operatorname{symb} \widehat{g}=(f * g)(\phi), \\
(f * g)(\phi)=f(\phi) \exp \left(-\frac{i \hbar}{2} \overleftarrow{\partial}_{a} \omega^{a b} \vec{\partial}_{b}\right) g(\phi)
\end{gathered}
$$

and the Moyal bracket, which is the symbol corresponding to the commutator

$$
\{f, g\}_{m b}=\frac{1}{i \hbar}(f * g-g * f)=\operatorname{symb}\left(\frac{1}{i \hbar}[f, g]\right)
$$

so we have

$(f * g)(\phi)=f(\phi) g(\phi)+0(\hbar),\{f, g\}_{m b}=\{f, g\}_{p b}+0\left(\hbar^{2}\right)$

To obtain the inverse $s y m b^{-1}$ we will use the symmetrical or Weyl ordering prescription, namely

$$
\operatorname{symb}^{-1}\left[q^{i}(\phi) p^{j}(\phi)\right]=\frac{1}{2}\left(\widehat{q}^{i} \widehat{p}^{j}+\widehat{p}^{j} \widehat{q}^{i}\right)
$$

Then we have an isomorphism between the quantum algebra $\widehat{\mathcal{A}}$ and the classical one $\mathcal{A}$

$$
\operatorname{symb}^{-1}: \mathcal{A} \rightarrow \widehat{\mathcal{A}}, \quad \int+\widehat{\mathbb{L} L}: \widehat{\mathcal{A}} \rightarrow \mathcal{A}
$$

The mapping so defined is the Weyl-Wigner-Moyal symbol.

For the state we have

$$
\rho(\phi)=\operatorname{symb} \widehat{\rho}=(2 \pi \hbar)^{-N-1} \operatorname{symb}_{(\text {for operators })} \widehat{\rho}
$$

and it turns out that

$$
(\widehat{\rho} \mid \widehat{O})=(\operatorname{symb} \widehat{\rho} \mid \text { symb } \widehat{O})=\int d \phi^{2(N+1)} \rho(\phi) O(\phi)
$$

Namely the definition $\widehat{\rho} \epsilon \widehat{\mathcal{A}^{\prime}}$, as afunctional on $\widehat{\mathcal{A}}$, is equal to the definition symb $\rho \in \mathcal{A}^{\prime}$, as afunctional on $\mathcal{A}$. 


\section{Decoherence in non integrable sys- tems}

\subsection{Local CSCO.}

a.- When our quantum system is endowed with a CSCO of $N+1$ observables, containing $\widehat{H}$, the underlying classical system is integrable. In fact, let $N+1-\mathrm{CSCO}$ be $\left\{\widehat{H}, \widehat{O}_{1}, \ldots, \widehat{O}_{N}\right\}$ the Moyal brackets of these quantities are

$$
\left\{O_{I}(\phi), O_{J}(\phi)\right\}_{m b}=\operatorname{symb}\left(\frac{1}{i \hbar}\left[\widehat{O}_{I}, \widehat{O}_{J}\right]\right)=0
$$

where $I, J, \ldots=0,1, \ldots, N$ and $\widehat{H}=\widehat{O}_{0}$. Then when $\hbar \rightarrow 0$ from Eq.(1) we know that

$$
\left\{O_{I}(\phi), O_{J}(\phi)\right\}_{p b}=0
$$

then as $H(\phi)=O_{0}(\phi)$ the set $\left\{O_{I}(\phi)\right\}$ is a complete set of $N+1$ constants of the motion in involution, globally defined over all $\mathcal{M}$, and therefore the system is integrable. q. e. $d$.

b.- If this is not the case $N+1$ constants of the motion in involution $\left\{H, O_{1}, \ldots, O_{N}\right\}$ always exist locally, as can be shown integrating the system of equations (3). Then, if $\phi_{i} \in \mathcal{M}$ there is maximal domain of integration $\mathcal{D}_{\phi_{\rangle}}$around $\phi_{i} \in \mathcal{M}$ where these constants are defined. In this case the system in non-integrable. Moreover we can repeat the procedure with the system

$$
\left\{O_{I}(\phi), O_{J}(\phi)\right\}_{m b}=0
$$

Then we can extend the definition of the constant $\left\{H, O_{1}, \ldots, O_{N}\right\}$, defined in each $\mathcal{D}_{\phi\rangle}$, outside $\mathcal{D}_{\phi\rangle}$ as null functions. Their Weyl transforms $\left\{\widehat{H}, \widehat{O}_{1}, \ldots, \widehat{O}{ }_{N}\right\}$ can be considered as a local $N+1$-CSCOs related each one with a domain $\mathcal{D}_{\phi_{\rangle}}$that we will call $\left\{\widehat{H}, \widehat{O}_{1 \phi_{i}}, \ldots, \widehat{O}{ }_{N \phi_{i}}\right\}$ (we consider that $\widehat{H}$ is always globally defined).

c.-We also can define an ad hoc positive partition of the identity

$$
1=I(\phi)=\sum_{i} I_{\phi_{i}}(\phi)
$$

where $I_{\phi_{i}}(\phi)$ is the characteristic function or index function, i.e.:

$$
I_{\phi_{i}}(\phi)=\left\{\begin{array}{l}
1 \text { if } \phi \epsilon D_{\phi_{i}} \\
0 \text { if } \phi \notin D_{\phi_{i}}
\end{array}\right.
$$

where the domains $D_{\phi_{i}} \subset \mathcal{D}_{\phi_{\rangle}} D_{\phi_{i}} \cap D_{\phi_{j}}=\emptyset$. Then $\sum_{i} I_{\phi_{i}}(\phi)=1$. Then we can define $A_{\phi_{i}}(\phi)=A(\phi) I_{\phi_{i}}(\phi)$ and

$$
A(\phi)=\sum_{i} A_{\phi_{i}}(\phi)
$$

and using $s y m b^{-1}$

$$
\widehat{A}=\sum_{i} \widehat{A}_{\phi_{i}}
$$

We can further decompose

$$
\widehat{A}_{\phi_{i}}=\sum_{j} A_{j \phi_{i}}|j\rangle_{\phi_{i}}\left\langle\left. j\right|_{\phi_{i}}\right.
$$

where the $|j\rangle_{\phi_{i}}$ are the corresponding eigenvectors of the local $N+1-\mathrm{CSCO}$ of $D_{\phi_{i}} \subset \mathcal{D}_{\phi_{\rangle}}$where a local $N+1-\mathrm{CSCO}$ is defined.. So

$$
\widehat{A}=\sum_{i j} A_{j \phi_{i}}|j\rangle_{\phi_{i}}\left\langle\left. j\right|_{\phi_{i}}\right.
$$

all over $\mathcal{M}$. It can be proved that for $i \neq k$ it is

$$
\left\langle\left. j\right|_{\phi_{i}} \mid j\right\rangle_{\phi_{k}}=0
$$

so the last decomposition is orthonormal, thus decomposition (5) generalizes the usual eigen-decomposition of integrable system to the non-integrable case. We will use this decomposition below.

\subsection{Decoherence in the energy.}

a.- Let us define in each $D_{\phi_{i}}$ a local $N+1-\operatorname{CSCO}\{\widehat{H}$ , $\left.\widehat{O_{\phi_{i}}}\right\}$ (as we have said we consider that $\widehat{H}$ is always globally defined) as

$$
\begin{aligned}
\widehat{H} & =\int_{0}^{\infty} \omega \sum_{i m}|\omega, m\rangle_{\phi_{i}}\left\langle\omega,\left.m\right|_{\phi_{i}} d \omega,\right. \\
\widehat{O_{\phi_{i} I}} & =\int_{0}^{\infty} \sum_{m} O_{m_{I \phi_{i}}}|\omega, m\rangle_{\phi_{i}}\left\langle\omega,\left.m\right|_{\phi_{i}} d \omega\right.
\end{aligned}
$$

where we have used decomposition (5). The energy spectrum is $0 \leq \omega<\infty$ and $m_{I \phi_{i}}=$ $\left\{m_{1 \phi_{i}}, \ldots, m_{N \phi_{i}}\right\}, m_{I \phi_{i}} \in \mathbb{N}$. Therefore

$$
\widehat{H}|\omega, m\rangle_{\phi_{i}}=\omega|\omega, m\rangle_{\phi_{i}}, \widehat{O_{\phi_{i} I}}|\omega, m\rangle_{\phi_{i}}=O_{m_{I \phi_{i}}}|\omega, m\rangle_{\phi_{i}}
$$

where, from the orthonormality of the eigenvector and Eq.(5), we have

$$
\left\langle\omega,\left.m\right|_{\phi_{i}} \mid \omega^{\prime}, m^{\prime}\right\rangle_{\phi_{j}}=\delta\left(\omega-\omega^{\prime}\right) \delta_{m m^{\prime}} \delta_{i j}
$$

b.- A generic observable, in the orthonormal basis just defined, reads:

$\widehat{O}=\sum_{i m m^{\prime}} \int_{0}^{\infty} \int_{0}^{\infty} d \omega d \omega^{\prime} \widetilde{O}\left(\omega, \omega^{\prime}\right)_{\phi_{i} m m^{\prime}}|\omega, m\rangle_{\phi_{i}}\left\langle\omega^{\prime},\left.m^{\prime}\right|_{\phi_{i}}\right.$

where $\widetilde{O}\left(\omega, \omega^{\prime}\right)_{\phi_{i} m m^{\prime}}$ is a generic kernel or distribution in $\omega, \omega^{\prime}$. As explained in the introduction, the simplest choice to solve our problem is the van Hove choice [4].

$\widetilde{O}\left(\omega, \omega^{\prime}\right)_{\phi_{i} m m^{\prime}}=O(\omega)_{\phi_{i} m m^{\prime}} \delta\left(\omega-\omega^{\prime}\right)+O\left(\omega, \omega^{\prime}\right)_{\phi_{i} m m^{\prime}}$

where we have a singular and a regular term, so called because the first one contains a Dirac delta and in the second one the $O\left(\omega, \omega^{\prime}\right)_{\phi_{i} m m^{\prime}}$ are ordinary functions of the real variables $\omega$ and $\omega^{\prime}$. As we will see these two parts appear in every formulae below. So our operators belong to an algebra $\widehat{\mathcal{A}}$ and they read

$$
\widehat{O}=\sum_{i m m^{\prime}} \int_{0}^{\infty} d \omega O(\omega)_{\phi_{i} m m^{\prime}}|\omega, m\rangle_{\phi_{i}}\left\langle\omega,\left.m^{\prime}\right|_{\phi_{i}}+\right.
$$




$$
\sum_{i m m^{\prime}} \int_{0}^{\infty} \int_{0}^{\infty} d \omega d \omega^{\prime} O\left(\omega, \omega^{\prime}\right)_{\phi_{i} m m^{\prime}}|\omega, m\rangle_{\phi_{i}}\left\langle\omega^{\prime},\left.m^{\prime}\right|_{\phi_{i}}\right.
$$

The observables are the self adjoint $O^{\dagger}=O$ operators. These observables belong to a space $\widehat{\mathcal{O}} \subset \widehat{\mathcal{A}}$. This space has the basis $\left.\left.\left\{\mid \omega, m, m^{\prime}\right)_{\phi_{i}}, \mid \omega, \omega^{\prime}, m, m^{\prime}\right)_{\phi_{i}}\right\}$ defined as:

$$
\begin{aligned}
\left.\mid \omega, m, m^{\prime}\right)_{\phi_{i}} & \doteq|\omega, m\rangle_{\phi_{i}}\left\langle\omega,\left.m^{\prime}\right|_{\phi_{i}},\right. \\
\left.\mid \omega, \omega^{\prime}, m, m^{\prime}\right)_{\phi_{i}} & \doteq|\omega, m\rangle_{\phi_{i}}\left\langle\omega^{\prime},\left.m^{\prime}\right|_{\phi_{i}}\right.
\end{aligned}
$$

c.- Let us define the quantum states $\widehat{\rho} \in \widehat{\mathcal{S}} \subset \widehat{\mathcal{O}}^{\prime}$, where $\widehat{\mathcal{S}}$ is a convex set. The basis of $\widehat{\mathcal{O}}^{\prime}$ is $\left\{\left(\omega,\left.m m^{\prime}\right|_{\phi_{i}},\left(\omega \omega^{\prime},\left.m m^{\prime}\right|_{\phi_{i}}\right\}\right.\right.$ and its vectors are defined as functionals by the equations:

$$
\begin{gathered}
\left(\omega, m,\left.m^{\prime}\right|_{\phi_{i}} \mid \eta, n, n^{\prime}\right)_{\phi_{j}}=\delta(\omega-\eta) \delta_{m n} \delta_{m^{\prime} n^{\prime}} \delta_{i j} \\
\left(\omega, \omega^{\prime}, m,\left.m^{\prime}\right|_{\phi_{i}} \mid \eta, \eta^{\prime}, n, n^{\prime}\right)_{\phi_{j}}= \\
\delta(\omega-\eta) \delta\left(\omega^{\prime}-\eta^{\prime}\right) \delta_{m n} \delta_{m^{\prime} n^{\prime}} \delta_{i j}
\end{gathered}
$$

and all others (.|.) are zero. Then, a generic quantum state reads:

$$
\begin{gathered}
\widehat{\rho}=\sum_{i m m^{\prime}} \int_{0}^{\infty} d \omega \overline{\rho(\omega)}_{\phi_{i} m m^{\prime}}\left(\omega,\left.m m^{\prime}\right|_{\phi_{i}}+\right. \\
\sum_{i m m^{\prime}} \int_{0}^{\infty} d \omega \int_{0}^{\infty} d \omega^{\prime} \overline{\rho\left(\omega, \omega^{\prime}\right)_{\phi_{i} m m^{\prime}}}\left(\omega \omega^{\prime},\left.m m^{\prime}\right|_{\phi_{i}}\right.
\end{gathered}
$$

We require that:

$$
\begin{aligned}
& {\overline{\rho\left(\omega, \omega^{\prime}\right)_{\phi_{i} m m^{\prime}}}}=\rho\left(\omega^{\prime}, \omega\right)_{\phi_{i} m^{\prime} m}, \\
& \rho(\omega, \omega)_{\phi_{i} m m} \geq 0, \\
& (\widehat{\rho} \mid \widehat{I})=\sum_{i m} \int_{0}^{\infty} d \omega \rho(\omega)_{\phi_{i}}=1,
\end{aligned}
$$

where $\widehat{I}=\int_{0}^{\infty} d \omega \sum_{i m}|\omega, m\rangle_{\phi_{i}}\left\langle\omega,\left.m\right|_{\phi_{i}}\right.$ is the identity operator. Then, in fact, $\widehat{\rho} \in \widehat{\mathcal{S}}$, where $\widehat{\mathcal{S}}$ is a convex set, and we have

$$
\begin{gathered}
\langle\widehat{O}\rangle_{\widehat{\rho}(t)=}(\widehat{\rho}(t) \mid \widehat{O})=\sum_{i m m^{\prime}} \int_{0}^{\infty} d \omega \overline{\rho(\omega)}_{\phi_{i} m m^{\prime}} O(\omega)_{\phi_{i} m m^{\prime}}+ \\
\sum_{i m m^{\prime}} \int_{0}^{\infty} d \omega \int_{0}^{\infty} d \omega^{\prime} \overline{\rho\left(\omega, \omega^{\prime}\right)}{ }_{\phi_{i} m m^{\prime}} \\
\quad \times e^{i\left(\omega-\omega^{\prime}\right) t / \hbar} O\left(\omega, \omega^{\prime}\right)_{\phi_{i} m m^{\prime}}
\end{gathered}
$$

If we now take the limit $t \rightarrow \infty$ and use the RiemannLebesgue theorem, being $O\left(\omega, \omega^{\prime}\right)$ and ${\overline{\rho\left(\omega, \omega^{\prime}\right)}}_{\phi_{i} m m^{\prime}}$ regu$\operatorname{lar}\left(\right.$ namely ${ }^{\prime}{\overline{\rho\left(\omega, \omega^{\prime}\right)}}_{\phi_{i} m m^{\prime}} O\left(\omega, \omega^{\prime}\right) \epsilon \mathbb{L}_{1}$ in the variable $\nu=$ $\left.\omega-\omega^{\prime}\right)$, we arrive to

$$
\begin{aligned}
& \lim _{t \rightarrow \infty}\langle\widehat{O}\rangle_{\widehat{\rho}(t)}=\left(\widehat{\rho}_{*} \mid \widehat{O}\right)= \\
& \sum_{i m m^{\prime}} \int_{0}^{\infty} d \omega \overline{\rho(\omega)}_{\phi_{i} m m^{\prime}} O(\omega)_{\phi_{i} m m^{\prime}}
\end{aligned}
$$

or to the weak limit

$W \lim _{t \rightarrow \infty} \widehat{\rho}(t)=\widehat{\rho}_{*}=\sum_{i m m^{\prime}} \int_{0}^{\infty} d \omega \overline{\rho(\omega)}_{\phi_{i} m m^{\prime}}\left(\omega, m,\left.m^{\prime}\right|_{\phi_{i}}\right.$

where only the diagonal-singular terms remain showing that the system has decohered in the energy.

\section{Remarks}

i.- It looks like that decoherence takes place without a coarse-graining, or an environment. It is not so, the van Hove choice (6) and the mean value (8) are a restriction of the information as effective as the coarse-graining is to produce decoherence.

ii.-Theoretically decoherence takes place at $t \rightarrow \infty$. Nevertheless, for atomic interactions, the characteristic decoherence time is $t_{D}=10^{-15} \mathrm{~s}$ [5]. For macroscopic systems this time is even smaller (e.g., $10^{-38} \mathrm{~s}$ ). Models with two characteristic times (decoherence and relaxation) can also be considered [6].

\subsection{Decoherence in the other variables.}

By a change of basis we can diagonalize the $\overline{\rho(\omega)}_{\phi_{i} m m^{\prime}}$ in $m$ and $m^{\prime}$ :

$$
\rho(\omega)_{\phi_{i} m m^{\prime}} \rightarrow \rho(\omega)_{\phi_{i} p p^{\prime}}=\rho_{\phi_{i}}(\omega)_{p} \delta_{p p^{\prime}} .
$$

in a new basis orthonormal $\left\{|\omega, p\rangle_{\phi_{i}}\right\}$. Therefore $\rho_{\phi_{i}}(\omega)_{p} \delta_{p p^{\prime}}$. is now diagonal in all its coordinates in a $f$ nal local pointer basis in each $D_{\phi_{i}}$, which, in the case of the observables is $\left.\left.\left\{\mid \omega, p, p^{\prime}\right)_{\phi_{i}}, \mid \omega, \omega^{\prime}, p, p^{\prime}\right)_{\phi_{i}}\right\}$ (i. e. essentially $\left\{\left|\omega^{\prime}, p^{\prime}\right\rangle_{\phi_{i}}\right\}$ ), so in this pointer basis we have obtained a boolean quantum mechanics with no interference terms and we have the weak limit:

$$
W \lim _{t \rightarrow \infty} \widehat{\rho}(t)=\widehat{\rho}_{*}=\sum_{i p} \int_{0}^{\infty} d \omega \overline{\rho_{\phi_{i}}(\omega)}{ }_{p}\left(\omega, p,\left.p\right|_{\phi_{i}}\right.
$$

or in the case of $\widehat{P}$ with continuous spectra:

$$
W \lim _{t \rightarrow \infty} \widehat{\rho}(t)=\widehat{\rho}_{*}=
$$

$$
\sum_{i} \int_{0}^{\infty} d \omega \int_{p \in D_{\phi_{i}}} d p^{N} \overline{\rho(\omega)_{\phi_{i}}}\left(\omega, p,\left.p\right|_{\phi_{i}}\right.
$$

the only case that we will consider below. 


\section{The classical statistical limit}

a.- Let us now take into account the Wigner transforms. There is no problem for regular operators which are considered in the standard theory. Moreover these operators are irrelevant since they disappear after decoherence.

b.- So we must only consider the singular ones as

$$
\widehat{O}_{S}=\sum_{i} \int_{p \in D_{\phi_{i}}} d p^{N} \int_{0}^{\infty} O_{\phi_{i}}(\omega, p)|\omega, p\rangle_{\phi_{i}}\left\langle\omega,\left.p\right|_{\phi_{i}} d \omega\right.
$$

where now the $\widehat{P}$ have continuous spectra. So

$$
\widehat{O}_{S}=\sum_{i} O_{\phi_{i}}\left(\widehat{H}, \widehat{P_{\phi_{i}}}\right)=\sum_{i} \widehat{O}_{S \phi_{i}}
$$

But $\widehat{H}, \widehat{P_{\phi_{i}}}$ commute thus

$$
\operatorname{symb} \widehat{O}_{S}=O_{S}(\phi)=\sum_{i} O_{\phi_{i}}\left(H(\phi), P_{\phi_{i}}(\phi)\right)+0\left(\hbar^{2}\right)
$$

and if $O_{\phi_{i}}(\omega, p)=\delta\left(\omega-\omega^{\prime}\right) \delta\left(p-p^{\prime}\right)$ we have

$$
\operatorname{symb}\left|\omega^{\prime}, p^{\prime}\right\rangle_{\phi_{i}}\left\langle\omega^{\prime},\left.p^{\prime}\right|_{\phi_{i}}=\delta\left(H(\phi)-\omega^{\prime}\right)\left(P_{\phi_{i}}(\phi)-p\right)\right.
$$

(really up to $0\left(\hbar^{2}\right)$, but for the sake of simplicity we will eliminate these symbols from now on).

Let us now consider the singular dual, the symb $\widehat{\rho}_{S}$ as the functional on $\mathcal{M}$ that must satisfy Eq.(2) that now reads

$$
\left(\operatorname{symb} \widehat{\rho}_{S} \mid \operatorname{symb} \widehat{O_{S}}\right)=\left(\widehat{\rho}_{S} \mid \widehat{O}_{S}\right)
$$

Then we define a density function $\rho_{S}(\phi)=\operatorname{symb} \widehat{\rho}_{S}$ $=\sum_{i} \rho_{\phi_{i} S}(\phi)$ such that

$$
\begin{gathered}
\sum_{i} \int d \phi^{2(N+1)} \rho_{\phi_{i} S}(\phi) O_{\phi_{i} S}(\phi)= \\
\sum_{i} \int_{p \in D_{\phi_{i}}} \int_{0}^{\infty} \rho_{\phi i}(\omega, p,) O_{\phi_{i}}(\omega, p) d \omega d p^{N}
\end{gathered}
$$

$\widehat{\rho_{S}}$, is constant of the motion, so $\rho_{\phi_{i}}(\phi)=$ $f\left(H(\phi), P_{\phi_{i}}(\phi)\right)$. Then we locally define at $D_{\phi_{i}}$ the local action-angle variables $\left(\theta^{0}, \theta^{1}, \ldots, \theta^{N}, J_{\phi_{i}}^{0}, J_{\phi_{i}}^{1}, \ldots, J_{\phi_{i}}^{N}\right)$, where $J_{\phi_{i}}^{0}, J_{\phi_{i}}^{1}, \ldots, J_{\phi_{i}}^{N}$ would just be $H, P_{\phi_{i} 1}, \ldots, P_{\phi_{i} N}$ and we make the canonical transformation $\phi^{a} \rightarrow$ $\theta_{\phi_{i}}^{0}, \theta_{\phi_{i}}^{1}, \ldots, \theta_{\phi_{i}}^{N}, H, P_{\phi_{i} 1}, \ldots, P_{\phi_{i} N}$ so that

$$
d \phi^{2(N+1)}=d q^{(N+1)} d p^{(N+1)}=d \theta_{\phi_{i}}^{(N+1)} d H d P_{\phi_{i}}^{N}
$$

Now we will integrate of the functions $f\left(H, P_{\phi_{i}}\right)=$ $f\left(H, P_{\phi_{i}}, \ldots, P_{\phi_{i}}\right)$ using the new variables.

$$
\begin{gathered}
\int_{D_{\phi_{i}}} d \phi^{2 N+2} f\left(H, P_{\phi_{i}}\right)=\int_{D_{\phi_{i}}} d \theta_{\phi_{i}}^{N+1} d H d P_{\phi_{i}}^{N} f\left(H, P_{\phi_{i}}\right) \\
=\int_{D \phi_{i}} d H d P_{\phi_{i}}^{N} C_{\phi_{i}}\left(H, P_{\phi_{i}}\right) f\left(H, P_{\phi_{i}}\right)
\end{gathered}
$$

where we have integrated the angular variables $\theta_{\phi_{i}}^{0}, \theta_{\phi_{i}}^{1}, \ldots, \theta_{\phi_{i}}^{N}$, obtaining the configuration volume
$C_{\phi_{i}}\left(H, P_{\phi_{i}}\right)$ of the portion of the hypersurface defined by ( $H=$ const., $P_{\phi_{i}}=$ const. $)$ and contained in $D_{\phi_{i}}$. So Eq.(10) reads

$$
\begin{gathered}
\sum_{i} \int_{p \in D_{\phi_{i}}} \int_{0}^{\infty} \rho_{\phi i}(\omega, p,) O_{\phi_{i}}(\omega, p) d \omega d p^{N}= \\
\sum_{i} \int d H d P_{\phi_{i}}^{N} C_{\phi_{i}}\left(H, P_{\phi_{i}}\right) \rho_{\phi_{i} S}\left(H, P_{\phi_{i}}\right) O_{\phi_{i} S}\left(H, P_{\phi_{i}}\right)
\end{gathered}
$$

for any $O_{\phi_{i}}(\omega, p)$ so $\rho_{S \phi_{i}}(H, P)=\frac{1}{C_{\phi_{i}}} \rho_{\phi_{i}}(H, P)$ for $\phi \epsilon \mathcal{D}_{\phi\rangle}$ and

$$
\rho_{S}(\phi)=\rho_{*}(\phi)=\sum_{i} \frac{\rho_{\phi_{i}}\left(H(\phi), P_{\phi_{i}}(\phi)\right)}{C_{\phi_{i}}\left(H, P_{\phi_{i}}\right)}
$$

Putting $\rho_{\phi_{i}}(\omega, p)=\delta\left(\omega-\omega^{\prime}\right) \delta^{N}\left(p-p^{\prime}\right)$ for some $i$ and all other $\rho_{\phi_{j}}(\omega, p)=0$ for $j \neq i$, we have

$$
\operatorname{symb}\left(\omega^{\prime}, p^{\prime},\left.(\phi)\right|_{\phi_{i}}=\frac{\delta\left(H(\phi)-\omega^{\prime}\right) \delta^{(N)}\left(P(\phi)-p_{\phi_{i}}^{\prime}\right)}{C_{\phi_{i}}\left(H, P_{\phi_{i}}\right)}\right.
$$

c.- Moreover the symb of Eq.(9) reads

$$
\rho_{S}(\phi)=\rho_{*}(\phi)=\sum_{i} \int_{p \epsilon D_{\phi_{i}}} d p \times
$$

$$
\int_{0}^{\infty} d \omega \rho_{\phi_{i}}(\omega, p) \frac{\delta(H(\phi)-\omega) \delta^{(N)}\left(P(\phi)-p_{\phi_{i}}\right)}{C_{\phi_{i}}\left(H, P_{\phi_{i}}\right)}
$$

So we have obtained a decomposition of $\rho_{*}(\phi)=$ $\rho_{S}(\phi)$ in classical hypersurfaces $\left(H=\omega, P_{\phi_{i}}(\phi)=\right.$ $p_{\phi_{i}}$ ), containing chaotic trajectories (since the system is not integrable), summed with different weight coefficients $\rho_{\phi_{i}}(\omega, p) / C_{\phi_{i}}\left(H, P_{\phi_{i}}\right)$.

d.- Finally only after decoherence the positive definite diagonal-singular part remains and from Eqs.(7) and (11) we see that

$$
\rho_{\phi_{i}}(\omega, p) \geq 0 \Rightarrow \rho_{*}(\phi) \geq 0
$$

so the classical statistical limit is obtained.

\section{The classical limit}

The classical limit can be decomposed into the following processes

$$
\text { Quantum Mechanics - (decohence) } \longrightarrow
$$

Boolean Quantum Mechanics-(symb and $\hbar \rightarrow 0) \longrightarrow$ Classical Statistical Mechanics-(choice of a trajectory) $\longrightarrow$ Classical Mechanics 
where the first two have been explained. It only remains the last one: For $\tau(\phi)=\theta_{\phi_{i}}^{0}(\phi)$ and at any fixed $t$ we have

$\sum_{i} \int_{D_{\phi_{i}}} \delta\left(\tau(\phi)-\tau_{0}-\omega t\right) \delta\left(\theta_{\phi_{i}}(\phi)-\theta_{\phi_{i} 0}-p_{\phi_{i}} t\right) d \tau_{0} d \theta_{\phi_{i} 0}=1$

then we can include this 1 in decomposition (11) and we obtain

$$
\begin{gathered}
\rho_{*}(\phi)=\sum_{i} \int \frac{\rho_{\phi_{i}}\left(\omega, p_{\phi_{i}}\right)}{C\left(\omega, p_{\phi_{i}}\right)} \delta(H(\phi)-\omega) \delta\left(P_{\phi_{i}}-p_{\phi_{i}}\right) \times \\
\delta\left(\tau(\phi)-\tau_{0}-\omega t\right) \delta\left(\theta_{\phi_{i}}(\phi)-\theta_{\phi_{i} 0}-p_{\phi_{i}} t\right) d \omega d^{N} p_{\phi_{i}} d \tau_{0} d \theta_{\phi_{i} 0}
\end{gathered}
$$

namely a sum of classical chaotic trajectories satisfying:

$$
\begin{gathered}
\left.H(\phi)=\omega, \quad \tau(\phi)=\tau_{0}+\omega t\right), \\
P_{\phi_{i}}=p_{\phi_{i}}, \quad \theta_{\phi_{i}}(\phi)=\theta_{\phi_{i} 0}+p_{\phi_{i}} t
\end{gathered}
$$

weighted by $\frac{\rho_{\phi_{i}}\left(\omega, p_{\phi_{i}}\right)}{C\left(\omega, p_{\phi_{i}}\right)}$,where we can choose any one of them. In this way the classical limit is completed, in fact we have found the classical limit of a quantum system since we have obtained the classical trajectories, so the correspondence principle is also obtained as a theorem.

\section{Conclusion}

i.- We have defined the classical limit in the non-integrable case. ii.- Essentially, we have presented a minimal formalism for quantum chaos [7].

iii.- We have deduced the correspondence principle.

\section{References}

[1] N.G. van Kampen, Physica XX, 603 (1954). A. Daneri, A. Loinger, and G.M. Prosperi, Nucl. Phys. 33, 297 (1962).

[2] J.P. Paz and W. Zurek, "Environment-induced decoherence and the transition from classical to quantum", arXiv: quant$\mathrm{ph} / 0010011$ (2000).

[3] M. Castagnino and R. Laura, Phys. Rev., A62, 022107 (2000). M. Castagnino, Physica A 335, 511 (2004). M. Castagnino and O. Lombardi, Stud. Phil. and Hist. Mod. Phys. 35, 73 (2004). M. Castagnino and O. Lombardi, "Self-Induced decoherence and the classical limit of quantum mechanics", Phil. of Sci., in press (2004).

[4] L. van Hove, Physica 21, 517 (1955); 23, 441 (1957); 25, 268 (1959).

[5] M. Castagnino, M. Gadella, R. Liotta, and R. Id Betan, Journ. Phys. A34, 10067 (2001). M. Castagnino, R. Laura, R. Liotta, and R. Id Betan, Journ. Phys. A35, 6055 (2002).

[6] M. Castagnino, "The master and Fokker-Plank equations of the self-induced approach", in preparation (2004).

[7] M. Castagnino, "Quantum chaos according to the self-induced approach", in preparation (2004). 